\title{
Making of Intelligent Simulation Experiment of Sound Velocity Measurement
}

\section{Jiang Xingfang ${ }^{1}$ 2* $^{*}$ Zhang Lei ${ }^{1}$, Lohani Md Badrul Alam ${ }^{1}$, Zhang Yuxin ${ }^{3}$}

\author{
${ }^{1}$ School of Microelectronics and control engineering, Changzhou University, \\ Changzhou, 213164, China \\ 2国家海洋局第二海洋研究所卫星海洋环境动力学国家重点实验室, 杭州 310012 \\ 3江苏省天一中学，无锡 214171 \\ E-mail: xfjiang@cczu.edu.cn; user.china@hotmail.com; \\ badrulalam.bdg@hotmail.com; 2990569285@qq.com
}

*Corresponding author details: Professor Jiang Xingfang; xfjiang@cczu.edu.cn

\begin{abstract}
Ultrasound is used as the wave source for the sound velocity measurement experiment. Ultrasonic wave is used to form standing wave in the reflection interval, the position of two adjacent wave anti nodes is used to determine the half wavelength, and the sound velocity in the experimental state is measured. The specific experimental apparatus applies a spiral structure. The hand-wheel rotates through one cycle and the ultrasound emitter moves $1 \mathrm{~mm}$ accordingly. Therefore, the circumference of the drum wheel driven by the hand-wheel is equally divided into 100 scales, each scale is for one grid, and a spin of one grid is one percent of $1 \mathrm{~mm}$, thus the accuracy can be $0.001 \mathrm{~mm}$. But there usually exists half pitch deviation because of the clockwise and counterclockwise rotation of the drum wheel clockwise and counterclockwise usually produces half pitch. For the sound velocity measurement experiment it is unacceptable to rotate the drum-wheel at different directions back and forth. In order to avoid this nuisance, we propose a computer-based simulation experiment system and this system can help to obtain a higher precision which is up to four significant digits when compared to the practical experiment. This simulation system can effectively avoid operational mistakes by the experimenter. The experimental data henceforth will be of ideal precision. This system is also capable of repetitive operation which can make up for the deficiency of practical experiments.
\end{abstract}

Keywords: intelligent system; experimental simulation; sound velocity measurement; unidirectional measurement

\section{INTRODUCTION}

The definition of velocity is the distance an object travels in a given time and the velocity of sound is an important physical quantity. Experience tells us the velocity of sound is $331.45 \mathrm{~m} / \mathrm{s}$ [1-2] when the temperature is $0^{\circ} \mathrm{C}$. In the laboratory, sound velocity is usually determined as the product of wavelength and frequency. The apparatus shown in FIGURE 1 consists of 3 parts: the signal source, sound velocity measurement instrument, and oscilloscope.

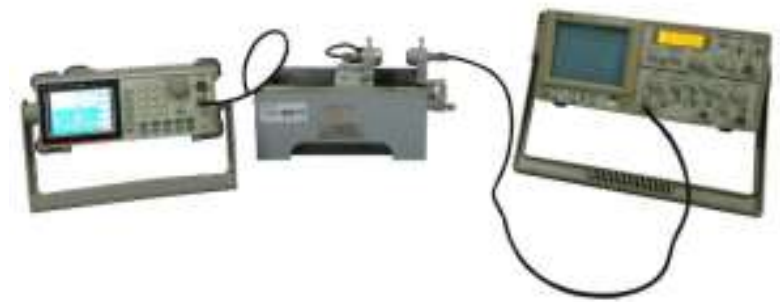

FIGURE 1: The apparatus of measurement
The frequency of the sound can be captured by a human is about $20 \sim 20000 \mathrm{~Hz}$, namely audible sound.

It is outrageous that dozens of apparatus emit audible sound simultaneously which will hinder our experiment. Thus, the sound source is set to be ultrasound. We devise the resonant frequency of emitter and receiver to be around $41 \mathrm{kHz}$ for the measurement at more than 10 periods, considering that ultrasound sound emitter can move in the range of $10 \mathrm{~cm}$. The sound velocity instrument usually applies the resonant interfering method and phase method to measure the velocity of sound in the laboratory environment.

The movable arm of the sound velocity measurement instrument is connected to the emitter, the signal source is then connected to the emitter, and the fixed arm linked to the receiver; the oscilloscope is also 
connected to the receiver. The hand-wheel rotates for one cycle and drives the drum-wheel shown in
FIGURE 2. spinning through one cycle. At the same time the screw powers the emitter to move $1 \mathrm{~mm}$.

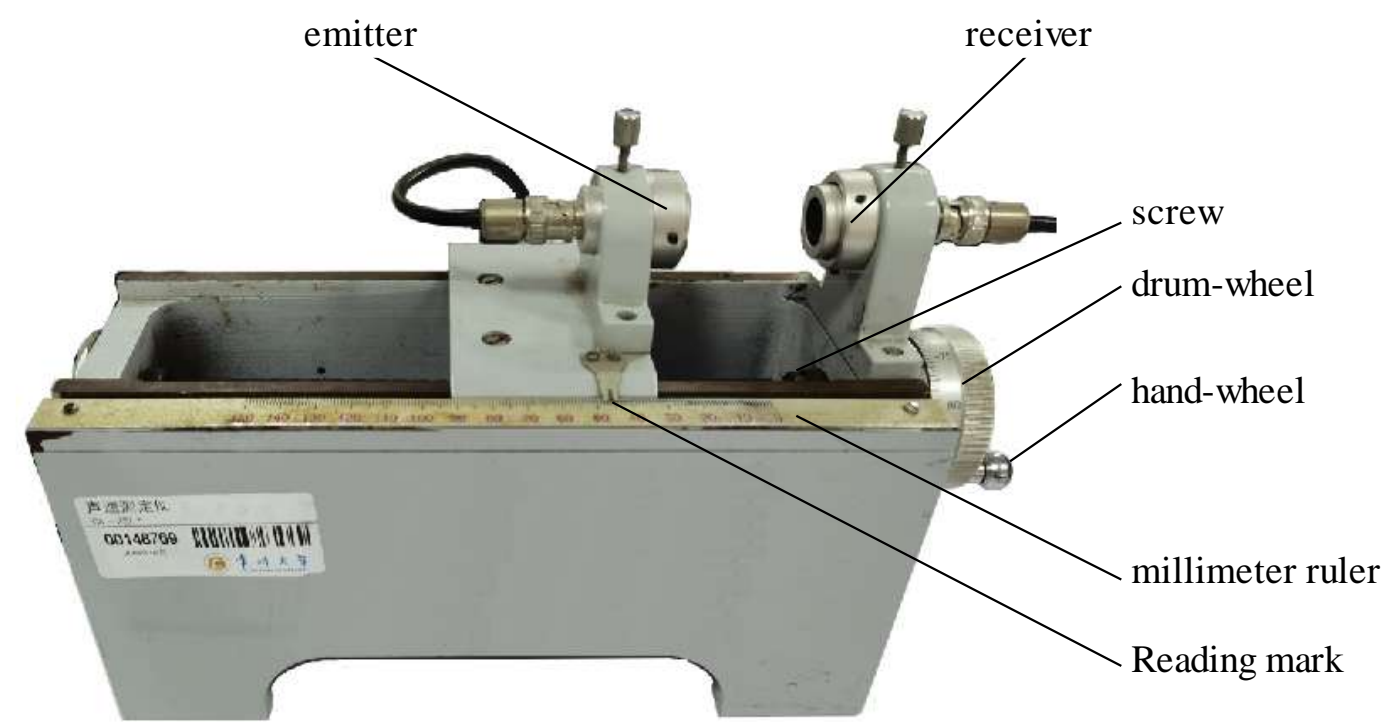

FIGURE 2: Sound velocity Instrument

When the screw rotates clockwise and counterclockwise it causes a statistical error of half of one pitch shown in FIGURE 3. and thus, the precise indication of the drum-wheel becomes meaningless. Therefore, we have to restrain the hand-wheel to rotate at just one direction ${ }^{[4]}$ during the reading.

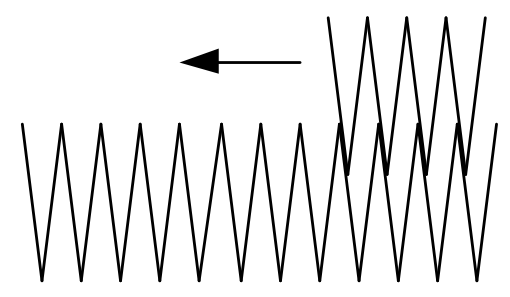

(a)

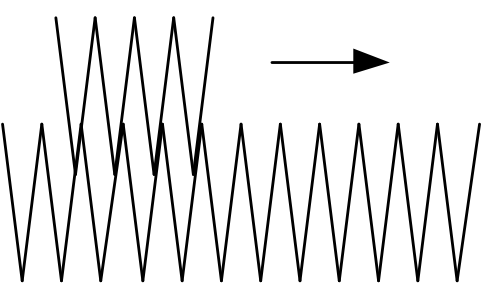

(b)

FIGURE 3: The motivation by screw

As shown above, the screw powers the emitter to move left in FIGURE 3. (a) and right in FIGURE 3. (b). While in our simulation system the travel direction is limited to left all the way.

\section{SIMULATION EXPERIMENT DESIGN}

The Flash CS6 is selected for our development tool and ActionScript 3.0 is as the programming language. The designing interface is shown as FIGURE 4. To highlight the dynamic part of the experiment we deploy two red dots and T-shape parts under the title. The left T-shape part is named 'emit', 'ball1' for the left dot, and 'ball2' for the right dot. Next, there are 3 fill able fields for 'Enter the student ID', 'Input temperature', and 'Input hand-wheel rotation Angle'. The instance names are 'field1', 'field2', and 'field3' respectively. After the temperature is filled, the system will display the theoretical sound velocity in the domain named 'field4'. Behind the scene, the system calculates the velocity by

$$
v=331.45 \sqrt{\frac{T}{273.15}}, T=t+273.15
$$

After the experimenter inputs the Student ID and clicks on the "emit" element, the system automatically determines the corresponding initial position of the "emit" element according to the Student ID. When click on one of the 45 display fields to obtain data the display field shows the waveform amplitude of "emit" at the determined position and the position coordinates of the "emit" element. The ultrasound frequency at about $41 \mathrm{kHz}$ regarding the Student ID is displayed in the ultrasound frequency display field (the instance name is "field5"); When the experimenters enters the hand-wheel rotation angle, for example, 50 degrees, then click the "Rotate by input Angle" button, the hand-wheel will rotate counterclockwise around the drum center by 50 degrees, and "ball1" is accordingly placed at the position by amplitude height. The horizontal position is consistent with the right side of "emit", and the "ball2" as a comparison also circles correspondingly. 


\section{Sound velocity measurement experimental simulation system}

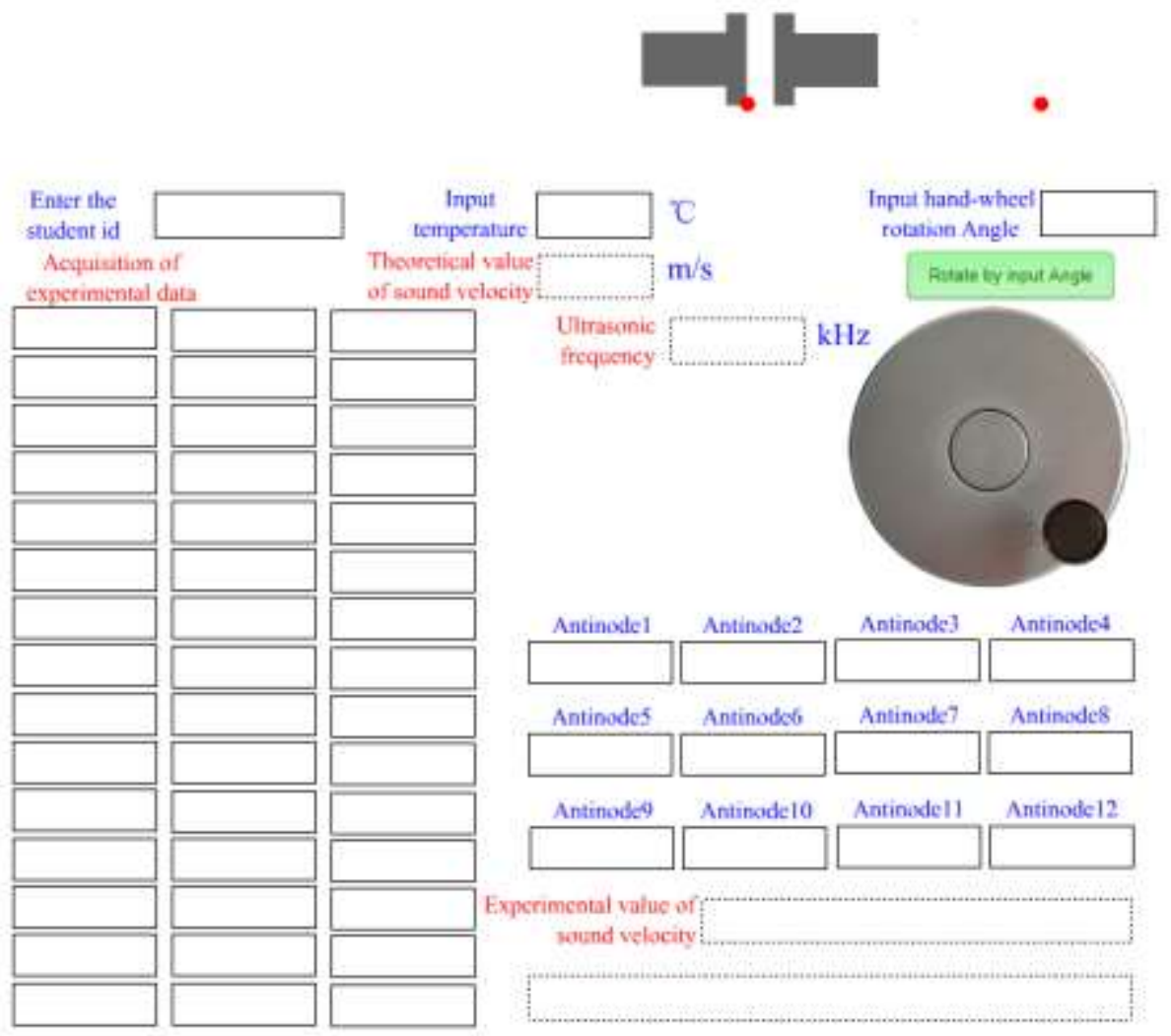

FIGURE 4: The Interface of the Simulation System

When the experimenter is on 45 display fields, their instance names are "field101", "field102", ..., "field145" respectively, the waveform amplitude at the determined "emit" position and the coordinate of the "emit" are displayed. After that, the experimenter is required to select the largest amplitude and fill it in "Anti node 1", and so on. After filling all 12 antinodes, click the field of sound velocity experimental value display (the instance name is "field6"), then the system will determine if the experimental data section is not fully filled and the calculated sound velocity is greater than $3 \%$ of the empirical value, it will prompt "The uncertainty is large, please do the simulation experiment again". If the experimental data section is fully filled and the simulation experiment uncertainty is lower $0.3 \%$, the result of the intelligent simulation experiment will have four significant digits.

\section{THE DEVELOPMENT OF SIMULATION EXPERIMENT SYSTEM}

Flash CS6 is an excellent intelligent software production system. Especially after the ActionScript 3.0 Language is used to code, the program structure is clearer. As an intelligent sound velocity measurement experiment simulation system, we have designed:

(1) When the experimenter inputs the local temperature, the system adopts

$$
v=331.45 \sqrt{\frac{T}{273.15}}, T=t+273.15 .
$$

The implementation of program is as below

var x1=Number (field3.text);

var $x 2=331.45 *$ Math.sqrt $((273.15+x 1) / 273.15)$;

field4.text=String $((\mathrm{x} 2 * 10-\mathrm{x} 2 * 10 \% 1) / 10)$;

if $((x 2 \% 1)>0.5)$ \{field4.text=String $((x 2 * 10+1-x 2 * 10 \% 1) / 10) ;\}$

Its function is to keep 1 decimal place to guarantee four significant digits.

(2) After the experimenter input the ID number and clicks "emit", the system reads the last three digits of the number when he. The code is

var x11=Number (field2.text); var x12=(x11/10) \% 1; var x13=x12*10 -(x12*10 \% 1);

var $\mathrm{x} 14$; var $\mathrm{x} 15$; $\operatorname{var} \mathrm{x} 16$; $\operatorname{var} \mathrm{x} 17$; $\operatorname{var} \mathrm{x} 18$;

if $((\mathrm{x} 12 * 10 \% 1)>0.5)\{\mathrm{x} 13=\mathrm{x} 12 * 10+1-(\mathrm{x} 12 * 10 \% 1) ;\}$

$\mathrm{x} 14=((\mathrm{x} 11-\mathrm{x} 13) / 100) \% 1$; 


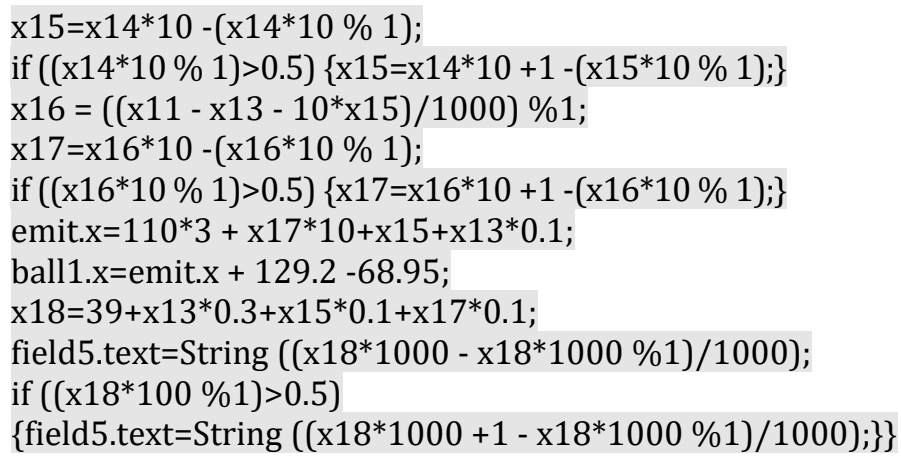

The last digit of the ID is $\mathrm{x} 14$, the penultimate is $\mathrm{x} 16$ and the third-to-last is $\mathrm{x} 18$. Furthermore, the $\mathrm{x} 14, \mathrm{x} 16, \mathrm{x} 18$ is a positive integer. The system determines the initial position of emitter by ID number and its varying range is $\pm 1 \mathrm{~mm}$. The ultrasound frequency will be differently obtained by different ID numbers and the range is $(41.5 \pm 1.5) \mathrm{kHz}$.

(3) After the experimenter inputs the angle at which

the hand-wheel rotates around the center of the drum and clicks the "Rotate by input angle" bar, the handwheel rotates around the center of the drum, and in the meantime, "ball1" and "ball2" also rotates at the corresponding angle. It is worthy the attention that the velocity of sound is related to the temperature, and the length of the experimental measurement wavelength is related to the frequency of the ultrasound wave, so the wavelength of the ultrasound wave is different according to the student ID number; even if the same experimenter performs simulation experiments at different temperatures, the ultrasound wavelength is also different. The program implementation is

var $\mathrm{x} 0=635.35$; $\operatorname{var} \mathrm{x} 01=663.65$; $\operatorname{var} \mathrm{y} 0=346.35$; $\operatorname{var} \mathrm{y} 01=101.6$;

var_R=Math.sqrt((635.35-689.30) * (635.35-689.30) + (346.35-399.55) * (346.35-399.55));

var $\mathrm{x} 1=$ Number (field1.text); var seta1; var seta2;

var lunx=lun.x; var ball2x=ball2.x; var luny=lun.y; var ball2y=ball2.y;

var x2=ball1.x; var y2=ball1.y; $\operatorname{var} \mathrm{x} 3=$ =mit.x; var $\mathrm{y} 3=$ =mit.y; $\operatorname{var} \mathrm{R} 1=54$;

var $\mathrm{x} 5=$ Number (field4.text); $\operatorname{var} \mathrm{x} 6=$ Number (field5.text); var $\mathrm{x} 7=\mathrm{x} 5 / \mathrm{x} 6$;

if (lunx-x0>0) \{seta1 = Math.atan $(($ luny-y0)/(lunx-x0))*180/3.14159;

if $($ seta $1<0)\{$ seta $1=$ seta $1+360 ;\}\}$

else $\{$ seta1=180+Math.atan((luny-y0)/(lunx-x0))*180/3.14159; $\}$

if (ball2x-x01>0) \{seta2=Math.atan((ball2y-y01)/(ball2x-x01))*180/3.14159;

if $($ seta2 $<0)\{$ seta $2=$ seta $2+360 ;\}\}$

else $\{$ seta2 $=180+$ Math.atan $(($ ball2y-y01)/(ball2x-x01) $) * 180 / 3.14159$;

lunx $=\mathrm{x} 0+\mathrm{R} *$ Math.cos(Number(seta1-x1)*3.14159/180);

luny $=\mathrm{y} 0+\mathrm{R}^{*}$ Math.sin(Number(seta $\left.\left.1-\mathrm{x} 1\right) * 3 \cdot 14159 / 180\right)$;

ball2x=x01+R1*Math.cos(Number(seta2-(x1/x7))*3.14159/180);

ball2y=y01-R1*Math.sin(Number(seta2-(x1/x7))*3.14159/180);

lun. $x=$ Number(lunx); lun. $y=$ Number(luny); ball2. $x=$ Number(ball2x); ball2. $y=$ Number(ball2y);

emit.x=emit. $\mathrm{x}$ x1*7.5/360; ball1.x=Number(x3+129.2 - 68.95);

ball1.y=Number(y01 - Math.abs(R1*Math.sin((seta2-x1/x7+0)*3.14159/180)));

if((seta2-x1)<0)\{ball1.y=Number(y01-Math.abs(R1*Math.sin((seta2-x1/x7+360)*3.14159/180)));

ball2.y=Number(y01 + (R1*Math.sin((seta2-x1/x7+0)*3.14159/180)));

(4) The experimenter clicks "field101" to display the vertical coordinate of "ball1" and the horizontal coordinate of the emitter "emit". The specific implementation method is

field101.addEventListener(MouseEvent.CLICK,F101);

function F101(e:MouseEvent):void\{var x1=ball1.y;

var x101=(434.35 - Number(emit.x))/75;

var $\mathrm{x} 201=(\mathrm{x} 101 * 1000-\mathrm{x} 101 * 1000 \% 1) / 1000$;

var $\mathrm{y} 101=101.25-\mathrm{x} 1$;

var y201=(y101*1000 - y101*1000\%1)/1000;

field101.text=String(y201+", "+x201);\}

The "field102", "field103", ..., "field145" will be made like "field101".

(5) The calculation of sound velocity

As shown in FIGURE 4, reading the amplitude of "ball1" and the position of "emit" at the lower left of the interface. When the amplitude of "ball1" reaches the maximum, the condition for standing wave formation is satisfied. The position where the amplitude reaches the maximum is the wave for the position of the antinode, the system designed 12 antinode positions to fill, the instance names are "field201", "field202", ..., "field212". The ultrasound wavelength value is calculated by the difference method, and then the wavelength and the product of the frequency is the wave velocity, the sound velocity is finally obtained. Then, the six sets of 1.5 times wavelength data obtained by the difference method are used to calculate the relative uncertainty as the uncertainty of the sound velocity. 
Finally, the experimental results of the sound velocity are displayed in "field6". The relative uncertainty of the obtained sound velocity and the empirical value is greater than $5 \%$, then the "field 90 " prompt field displays "Uncertainty is too large, do the simulation experiment again"; if the measured sound velocity and the empirical value are relatively uncertain if the degree is less than $0.3 \%$, only the experiment result of sound velocity will be displayed in "field6"; if it is between the two, the "field90" prompt field will display "the uncertainty is greater, and do the simulation experiment again". The specific procedures are as follows:

if (field212.text=="") $\{$ var x1=Number(field201.text); var x2=Number(field202.text); var x3=Number(field5.text); var v=2*Math.abs(x1-x2)/x3; var x4=Number(field4.text); var $\mathrm{x} 5$; var $\mathrm{x} 6=\mathrm{v}^{*} 10-\mathrm{v}^{*} 10 \% 1$;

if $\left(\left(\mathrm{v}^{*} 10 \% 1\right)>0.5\right) \quad\left\{\mathrm{x} 6=\mathrm{v}^{*} 10+1-\mathrm{v}^{*} 10 \% 1 ;\right\}$

$\mathrm{x} 5=\left(\mathrm{v}^{*} 10-\mathrm{x} 4\right) / \mathrm{x} 4$;

if(x5>0.05) \{field90.text="The uncertainty is too large, and do the simulation experiment again!";

else $\{$ field90.text=Number $(\mathrm{x} 6)+\mathrm{m} / \mathrm{s} " ;\}\}$

else $\{$ var x01=Math.abs(Number(field201.text)-Number(field207.text));

var x02=Math.abs(Number(field202.text)-Number(field208.text));

var x03=Math.abs(Number(field203.text)-Number(field209.text));

var $\mathrm{x} 04=$ =Math.abs(Number(field204.text)-Number(field210.text));

var $\mathrm{x} 05=$ =Math.abs(Number(field205.text)-Number(field211.text));

var x06=Math.abs(Number(field206.text)-Number(field212.text));

var $x a v g=(x 01+x 02+x 03+x 04+x 05+x 06) / 18$;

var deletex $=3 *$ Math.sqrt $(((\mathrm{x} 01-\mathrm{xavg}) *(\mathrm{x} 01-\mathrm{xavg})+(\mathrm{x} 02-\mathrm{xavg}) *(\mathrm{x} 02-\mathrm{xavg})+(\mathrm{x} 03-\mathrm{xavg}) *(\mathrm{x} 03-\mathrm{xavg})$

$+(\mathrm{x} 04-\mathrm{xavg}) *(\mathrm{x} 04-\mathrm{xavg})+(\mathrm{x} 05-\mathrm{xavg}) *(\mathrm{x} 05-\mathrm{xavg})+(\mathrm{x} 06-\mathrm{xavg}) *(\mathrm{x} 06-\mathrm{xavg})) / 30)$

var $\mathrm{x} 7=$ deletex/xavg; var $\mathrm{x} 8=$ Number(field5.text); var $\mathrm{x} 9=$ Number(field4.text);

var v1=xavg*x8*10; var $\mathrm{x} 10$; var $\mathrm{x} 11$;

if $(\mathrm{x} 7<0.9)\left\{\mathrm{x} 10=(\right.$ deletex*10 $+1-\operatorname{deletex} * 10 \% 1) / 10 ; \mathrm{x} 11=\left(\mathrm{v}^{*} 10+0-\mathrm{v} 1 * 10 \% 1\right) / 10$;

$\operatorname{if}\left(\left(\mathrm{v}^{*} 10 \% 1\right)>0.5\right)\{\mathrm{x} 11=(\mathrm{v} 1 * 10+1-\mathrm{v} 1 * 10 \% 1) / 10 ;\}$

field6.text="("+Number(x11)+" $\pm "+$ Number(x10)+")m/s";\}

else $\{$ field90.text=" The uncertainty is a little large, do the simulation experiment again!";

field6.text="("+ (v1 -v1 \% 1)+" $\pm "+($ deletex +1 - deletex \% 1)+")m/s";

if $\left(\left(x 7^{*} 100 \% 1\right)>0.5\right)\{$ field6.text="("+ (v1 +1 -v \% 1)+" $\pm "+($ deletex $\left.\left.\left.+1-\operatorname{deletex} \% 1)+") \mathrm{m} / \mathrm{s}^{\prime \prime} ;\right\}\right\}\right\}$

One of the experimental results is shown FIGURE 5 . and the obtained velocity value is $354 \pm 3 \mathrm{~m} / \mathrm{s}$ which has a difference of $1 \%$ of the empirical value. Basically, the simulation experiment can be more accurate to make the comparative uncertainty under $0.3 \%$.

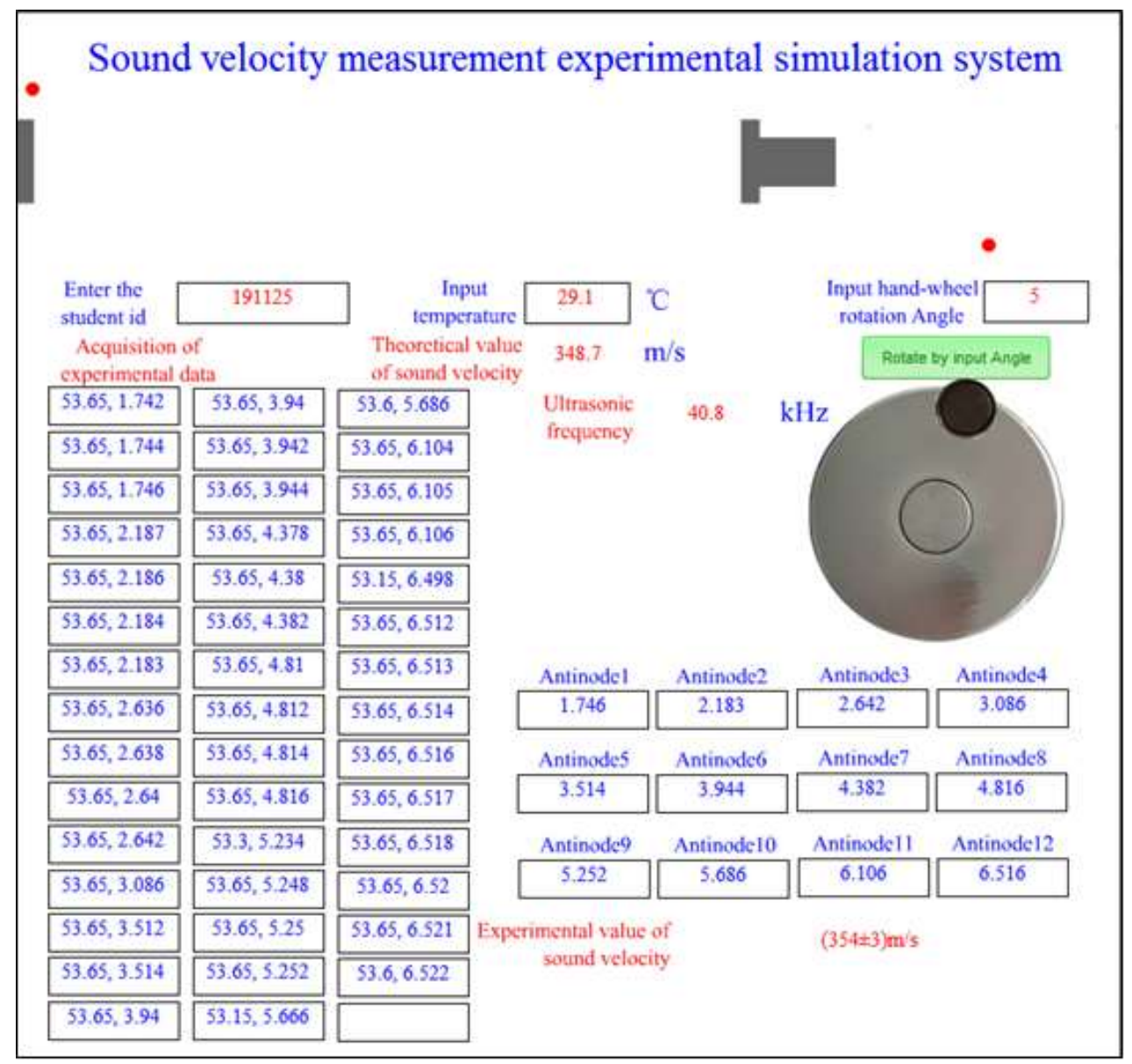

FIGURE 5: One of the experimental results 


\section{THE REFLECTION ON THE TEST OF THE EXPERIMENT}

The above experiment is very difficult for students to achieve excellence, and then consider that as the distance between the transmitter and the receiver increases, the amplitude decreases accordingly.
From the experimental results of [2], the amplitude varies with the distance between the transmitter and the receiver. It shows the distance decays exponentially. In the program is used for decaying. From Table 1, it can be estimated that the value of is 0.94228 .

TABLE 1: The relative amplitude of the abdominal position of each wave

\begin{tabular}{cccccccc}
\hline $\boldsymbol{x}$ & $\mathbf{0}$ & $0.5 \lambda$ & $1.0 \lambda$ & $1.5 \lambda$ & $2.0 \lambda$ & $2.5 \lambda$ & $3.0 \lambda$ \\
\hline $\operatorname{Amp} R_{11}$ & 111 & 89 & 38 & 20 & 15 & 10 & 8 \\
$\operatorname{Ln}\left(R_{11}\right)$ & 4.7095 & 4.4886 & 3.6376 & 2.9957 & 2.7081 & 2.3026 & 2.0794 \\
\hline
\end{tabular}

After substituting for it, the difficulty of obtaining excellent in experiments is greater that is closer to the real ideal experiment result.

\section{REFERENCE}

[1] Dennis A. Bohn. Environmental effects on the velocity of sound [J]. Journal Audio Engineer Society. 1987, 36(4): 1-9

[2] Bernard S. Finn. Laplace and the velocity of sound [J]. ISIS. 1964, 55(179): 7:19

[3] Jiang Xingfang. Three points in sound velocity measurement experiment [J]. College Physics. 2002. 21(12): 28-30+13 (in Chinese)

[4] Song Zi-wu, Wang Mao-xiang. Observation and analysis of abnormal phenomena in sound velocity measurement experiment [J]. College Physics. 2018, 37(11): 41-45 (in Chinese)
[5] Jiang Xingfang, Xie Jiansheng, Tang Li. Physical experiment (2nd Ed.). Beijing: Science Press. 2019 (in Chinese)

[6] Ye Yong. Design and implementation of sound velocity measurement simulation experiment based on Virtools [J]. Computer Mystery. 2018, 31: 121 (in Chinese)

[7] Hao Jin-bo, Xu Yang-bin, $\mathrm{Wu} \mathrm{Ge}$, et al. Sound velocity measurement simulation and data processing based on MATLAB GUI [J]. University Physics Experiment. 2019, 32(4): 79-83 (in Chinese) 